\title{
Real spectra in non-Hermitian topological insulators
}

\author{
Kohei Kawabata, ${ }^{1, *}$ and Masatoshi Sato ${ }^{2}$ \\ ${ }^{1}$ Department of Physics, University of Tokyo, 7-3-1 Hongo, Bunkyo-ku, Tokyo 113-0033, Japan \\ ${ }^{2}$ Yukawa Institute for Theoretical Physics, Kyoto University, Kyoto 606-8502, Japan
}

(Received 4 April 2020; accepted 18 August 2020; published 10 September 2020)

\begin{abstract}
Spectra of bulk or edges in topological insulators are often made complex by non-Hermiticity. Here, we show that symmetry protection enables entirely real spectra for both bulk and edges even in non-Hermitian topological insulators. In particular, we demonstrate the entirely real spectra without non-Hermitian skin effects due to a combination of pseudo-Hermiticity and Kramers degeneracy. This protection relies on nonspatial fundamental symmetry and has stability against disorder. As an illustrative example, we investigate a non-Hermitian extension of the Bernevig-Hughes-Zhang model. The helical edge states exhibit oscillatory dynamics due to their nonorthogonality as a unique non-Hermitian feature.
\end{abstract}

DOI: 10.1103/PhysRevResearch.2.033391

\section{INTRODUCTION}

Physics of non-Hermitian systems has generated considerable recent research interest [1,2]. Non-Hermiticity appears, for example, in open classical [3-13] and quantum [14-23] systems as a consequence of the external environment. Despite non-Hermiticity, Hamiltonians can have entirely real spectra if parity-time symmetry [24] or pseudo-Hermiticity [25] is respected. Disorder can also give rise to the real spectra in time-reversal-invariant non-Hermitian systems [26]. The reality of the spectra ensures the stability of the systems even in the presence of non-Hermiticity. On the other hand, when non-Hermiticity is sufficiently strong, the symmetry is spontaneously broken and some eigenenergies form complexconjugate pairs. An exceptional point appears between the two phases at which the eigenstates coalesce with each other [27]. The real spectra and exceptional points were experimentally observed in a number of classical and quantum systems, such as a photonic lattice [8], a microcavity [10], single photons [19], a nitrogen-vacancy center [20], and superconducting qubits [22].

Much research in recent years has focused on topological characterization of non-Hermitian systems [28,29] both in theory [30-69] and in experiments [70-83]. Non-Hermiticity alters the fundamental nature of the topological classification of phases of matter $[43,56,59]$ and the bulk-boundary correspondence $[35,44,46,49,55,60]$. Furthermore, the interplay of non-Hermiticity and topology leads to unique phenomena and functionalities that have no counterparts in conventional systems. A prime example is topological lasers [75-77,79]. Because of the judicious designs, they possess the real spectra

\footnotetext{
*kawabata@cat.phys.s.u-tokyo.ac.jp

Published by the American Physical Society under the terms of the Creative Commons Attribution 4.0 International license. Further distribution of this work must maintain attribution to the author(s) and the published article's title, journal citation, and DOI.
}

for the bulk but the complex spectra for the edges; whereas the bulk states remain stable, the edge states are amplified, resulting in high-efficiency lasers protected by topology.

Despite the significance of the reality of spectra, Ref. [32], which is one of the earliest works on non-Hermitian topological systems [30,31,32], showed that entirely real spectra of both bulk and edges are impossible in a large class of non-Hermitian topological insulators with parity-time symmetry. For example, when we introduce balanced gain and loss to the Su-Schrieffer-Heeger model [84] without breaking chiral symmetry (pseudo-anti-Hermiticity), the bulk spectrum remains real, but a pair of zero-energy edge states acquires nonzero imaginary eigenenergies $[31,33,73,75]$. On the other hand, when we introduce asymmetric hopping to the Su-Schrieffer-Heeger model [84] without breaking sublattice symmetry, the entirely real spectrum for both bulk and edges can be realized under the open boundary conditions $[35,44,46]$; however, it relies on the non-Hermitian skin effect and the spectrum becomes complex under the periodic boundary conditions. Remarkably, Ref. [32] assumes no symmetry other than parity-time symmetry and mentions possible exceptions of its theorem due to particle-hole or point-group symmetry. In fact, a $p$-wave topological superconducting wire with balanced gain and loss, which is described by a nonHermitian extension of the Kitaev chain [85] with parity-time symmetry, can possess the entirely real spectrum even in the presence of Majorana edge states [36,39,42]. By contrast, non-Hermitian topological insulators with entirely real spectra have yet to be known. Although the reality of spectra is relevant to the stability of non-Hermitian systems, the real spectra in non-Hermitian topological insulators have still been elusive.

In this work, we show that symmetry protection enables the entirely real spectra for both bulk and edges even in non-Hermitian topological insulators. This protection is due to nonspatial symmetry and stable against disorder. In Sec. II B, we demonstrate that generic time-reversal-invariant topological insulators in two dimensions can have real spectra even in the presence of non-Hermiticity as long as reciprocity 
(a variant of time-reversal symmetry in non-Hermitian systems) and pseudo-Hermiticity are respected. As shown in Sec. III with a continuum Dirac Hamiltonian, the discussions in Ref. [32] are not directly applicable because of additional pseudo-Hermiticity and reciprocity. As an illustrative example, we investigate a non-Hermitian extension of the Bernevig-Hughes-Zhang (BHZ) model [86] in Sec. IV. We explicitly show that it indeed has a real spectrum by both numerical and analytical calculations. Despite the real spectrum, it shows phenomena unique to non-Hermitian systems. In particular, the helical edge states exhibit oscillatory dynamics since they are nonorthogonal, as shown in Sec. V. We conclude this work in Sec. VI. In the Appendix, we investigate another non-Hermitian extension of the BHZ model that is protected by time-reversal symmetry and possesses the complex edge spectrum.

\section{REAL SPECTRA DUE TO SYMMETRY PROTECTION}

\section{A. Symmetry and topology}

We begin with a generic Hermitian Hamiltonian $H(\boldsymbol{k})$ in two dimensions that respects time-reversal symmetry:

$$
\mathcal{T} H^{*}(\boldsymbol{k}) \mathcal{T}^{-1}=H(-\boldsymbol{k}), \quad \mathcal{T} \mathcal{T}^{*}=-1,
$$

where $H(\boldsymbol{k})$ is a Bloch Hamiltonian, and $\mathcal{T}$ is a unitary matrix (i.e., $\mathcal{T} \mathcal{T}^{\dagger}=\mathcal{T}^{\dagger} \mathcal{T}=1$ ). The topological phase of $H(\boldsymbol{k})$ is characterized by the $\mathbb{Z}_{2}$ invariant, which induces the quantum spin Hall effect accompanying helical edge states [86-88]. Moreover, we consider additional unitary symmetry,

$$
\eta H(\boldsymbol{k}) \eta^{-1}=H(\boldsymbol{k}), \quad \eta^{2}=1,
$$

where $\eta$ is a unitary and Hermitian matrix (i.e., $\eta \eta^{\dagger}=\eta^{\dagger} \eta=$ 1). We assume that these symmetry anticommutes with each other:

$$
\mathcal{T} \eta^{*}=-\eta \mathcal{T}
$$

For example, the BHZ model [86] respects these symmetry in Eqs. (1), (2), and (3) with $\mathcal{T}=i \sigma_{y}$ and $\eta=\sigma_{z}$ :

$$
\begin{aligned}
H_{\mathrm{BHZ}}(\boldsymbol{k})= & \left(m+t \cos k_{x}+t \cos k_{y}\right) \tau_{z} \\
& +t\left(\sin k_{y}\right) \tau_{y}+t\left(\sin k_{x}\right) \sigma_{z} \tau_{x} .
\end{aligned}
$$

Here, Pauli matrices $\sigma_{i}$ 's and $\tau_{i}$ 's $(i=x, y, z)$ describe the spin and orbital degrees of freedom, respectively. The BHZ model describes mercury telluride-cadmium telluride semiconductor quantum wells that host the quantum spin Hall effect, in which the unitary symmetry in Eq. (2) represents the conservation of spin.

As a non-Hermitian generalization of these symmetry, we consider a generic non-Hermitian Hamiltonian $H(\boldsymbol{k})$ in two dimensions that respects

$$
\begin{gathered}
\mathcal{T} H^{T}(\boldsymbol{k}) \mathcal{T}^{-1}=H(-\boldsymbol{k}), \quad \mathcal{T} \mathcal{T}^{*}=-1, \\
\eta H^{\dagger}(\boldsymbol{k}) \eta^{-1}=H(\boldsymbol{k}), \quad \eta^{2}=1,
\end{gathered}
$$

where unitary matrices $\mathcal{T}$ and $\eta$ anticommute with each other [Eq. (3)]. Here, Eqs. (5) and (6) reduce to Eqs. (1) and (2) in the presence of Hermiticity [i.e., $H^{\dagger}(\boldsymbol{k})=H(\boldsymbol{k})$ ], respectively. When Eq. (5) is satisfied, the scattering matrix $S$ respects $\mathcal{T} S^{T} \mathcal{T}^{-1}=S$, and hence the scattering processes are reciprocal [89]. For example, an incoming spin-up wave is related to an outgoing spin-down wave because of $\mathcal{T} S^{T} \mathcal{T}^{-1}=S$. Consequently, Eq. (5) describes reciprocity in non-Hermitian systems and is relevant, for example, in mesoscopic systems [89] and open quantum systems [90-92]. It is also notable that this symmetry is a variant of time-reversal symmetry and called "TRS ${ }^{\dagger}$ " in Ref. [56]. On the other hand, Eq. (6) denotes pseudo-Hermiticity [25], which can lead to the real spectra of non-Hermitian systems (see Sec. II B for details). These symmetry is included in the 38 -fold internal symmetry in non-Hermitian physics [56,93]. Examples of the symmetry operators $\mathcal{T}$ and $\eta$ are given in the subsequent sections [see Eqs. (17) and (18)].

The $\mathbb{Z}_{2}$ topological phase survives non-Hermiticity as long as reciprocity in Eq. (5) is respected and the gap for the real part of eigenenergies remains open (i.e., $\forall \boldsymbol{k} \operatorname{Re} E(\boldsymbol{k}) \neq 0$; real line gap in Ref. [56]). Furthermore, even a $\mathbb{Z}$ topological invariant is well defined in the presence of additional pseudoHermiticity in Eq. (6). To see this $\mathbb{Z}$ invariant, let us focus on a matrix $\eta H(\boldsymbol{k})$. Because of pseudo-Hermiticity in Eq. (6), $\eta H(\boldsymbol{k})$ is Hermitian:

$$
[\eta H(\boldsymbol{k})]^{\dagger}=\eta H(\boldsymbol{k})
$$

In addition, $\eta H(\boldsymbol{k})$ has a gap when the original non-Hermitian Hamiltonian $H(\boldsymbol{k})$ has a gap for the real part of eigenenergies. Consequently, the Chern number is well defined for $\eta H(\boldsymbol{k})$, which characterizes the $\mathbb{Z}$ topological phase of $H(\boldsymbol{k})$. This is contrasted with the vanishing Chern number for $H(\boldsymbol{k})$ due to time-reversal symmetry (reciprocity). Notably, if reciprocity and pseudo-Hermiticity commute with each other (i.e., $\mathcal{T} \eta^{*}=$ $\eta \mathcal{T}$ ) instead of Eq. (3), $\eta H(\boldsymbol{k})$ respects time-reversal symmetry and its Chern number vanishes. The $\mathbb{Z}$ topological phases protected by reciprocity in Eq. (5) and pseudo-Hermiticity in Eq. (6) are consistent with the 38-fold classification of non-Hermitian topological phases (see Table IX in Ref. [56], with the symmetry class "AI $+\eta_{-}$" and two dimensions). The combination of reciprocity and pseudo-Hermiticity enables the well-defined $\mathbb{Z}$ invariant, while we only have a $\mathbb{Z}_{2}$ invariant if pseudo-Hermiticity is not respected (see also Table V in Ref. [56], with the symmetry class "AII ${ }^{\dagger}$ " and two dimensions). It is also remarkable that the $\mathbb{Z}$ invariant is equivalent to the time-reversal-invariant Chern number in Refs. [31,56].

\section{B. Real spectra}

A combination of the symmetry in Eqs. (5) and (6) leads to the entirely real spectra for both bulk and edges. The real spectra of the bulk are ensured by pseudo-Hermiticity in Eq. (6). To see this, let $E_{n}(\boldsymbol{k})$ be an eigenenergy of $H(\boldsymbol{k})$ and $\left|u_{n}(\boldsymbol{k})\right\rangle$ $\left.\left(\left|u_{n}(\boldsymbol{k})\right\rangle\right\rangle\right)$ be the corresponding right (left) eigenstate:

$$
\begin{aligned}
H(\boldsymbol{k})\left|u_{n}(\boldsymbol{k})\right\rangle & =E_{n}(\boldsymbol{k})\left|u_{n}(\boldsymbol{k})\right\rangle, \\
\left\langle\left\langle u_{n}(\boldsymbol{k})\right| H(\boldsymbol{k})\right. & =E_{n}(\boldsymbol{k})\left\langle\left\langle u_{n}(\boldsymbol{k})\right| .\right.
\end{aligned}
$$


In the presence of pseudo-Hermiticity in Eq. (6), we have

$$
\begin{aligned}
\left.H(\boldsymbol{k})\left[\eta\left|u_{n}(\boldsymbol{k})\right\rangle\right\rangle\right] & \left.=\eta H^{\dagger}(\boldsymbol{k})\left|u_{n}(\boldsymbol{k})\right\rangle\right\rangle \\
& \left.=E_{n}^{*}(\boldsymbol{k})\left[\eta\left|u_{n}(\boldsymbol{k})\right\rangle\right\rangle\right],
\end{aligned}
$$

which implies that $\left.\eta\left|u_{n}(\boldsymbol{k})\right\rangle\right\rangle$ is a right eigenstate of $H(\boldsymbol{k})$ with the eigenenergy $E_{n}^{*}(\boldsymbol{k})$. When non-Hermiticity is sufficiently weak, $\left|u_{n}(\boldsymbol{k})\right\rangle$ and $\left.\eta\left|u_{n}(\boldsymbol{k})\right\rangle\right\rangle$ should coincide with each other since they are the same single state in the absence of nonHermiticity. As a result, it holds

$$
E_{n}(\boldsymbol{k})=E_{n}^{*}(\boldsymbol{k}), \quad \text { i.e., } \quad E_{n}(\boldsymbol{k}) \in \mathbb{R} .
$$

On the other hand, when non-Hermiticity is strong enough to give rise to band touching, $\left|u_{n}(\boldsymbol{k})\right\rangle$ and $\left.\eta\left|u_{n}(\boldsymbol{k})\right\rangle\right\rangle$ are different, so the corresponding eigenenergies become complex in a pair. Thus, even in the presence of non-Hermiticity, an energy band with a real spectrum remains real as long as it is isolated from other bands and pseudo-Hermiticity is preserved. It can have a complex spectrum only if the energy gap is closed.

On the other hand, pseudo-Hermiticity alone does not necessarily lead to the real spectra of the boundary states. This is because the boundary states are gapless and hence can have complex spectra. Nevertheless, their reality can be ensured by reciprocity in Eq. (5). An important consequence of Eq. (5) is Kramers degeneracy $[31,56]$. To see this, we have

$$
\begin{aligned}
\left.H(\boldsymbol{k})\left[\mathcal{T}\left|u_{n}^{*}(-\boldsymbol{k})\right\rangle\right\rangle\right] & \left.=\mathcal{T} H^{T}(-\boldsymbol{k})\left|u_{n}^{*}(-\boldsymbol{k})\right\rangle\right\rangle \\
& \left.=E_{n}(-\boldsymbol{k})\left[\mathcal{T}\left|u_{n}^{*}(-\boldsymbol{k})\right\rangle\right\rangle\right],
\end{aligned}
$$

which implies that $\left.\mathcal{T}\left|u_{n}^{*}(-\boldsymbol{k})\right\rangle\right\rangle$ is a right eigenstate of $H(\boldsymbol{k})$ with the eigenenergy $E_{n}(-\boldsymbol{k})$. Hence, at a time-reversalinvariant momentum $\boldsymbol{k}_{\text {TRIM }}$ [i.e., $H\left(\boldsymbol{k}_{\text {TRIM }}\right)=H\left(-\boldsymbol{k}_{\text {TRIM }}\right)$ ], both $\left|u_{n}\left(\boldsymbol{k}_{\mathrm{TRIM}}\right)\right\rangle$ and $\left.\mathcal{T}\left|u_{n}^{*}\left(\boldsymbol{k}_{\mathrm{TRIM}}\right)\right\rangle\right\rangle$ belong to the same eigenenergy $E_{n}\left(\boldsymbol{k}_{\mathrm{TRIM}}\right)$. Moreover, because of $\mathcal{T}^{T}=-\mathcal{T}$, we have

$$
\begin{aligned}
\left\langle\left\langle u_{n}\left(\boldsymbol{k}_{\mathrm{TRIM}}\right)|\mathcal{T}| u_{n}\left(\boldsymbol{k}_{\mathrm{TRIM}}\right)\right\rangle\right\rangle & =\left\langle\left\langle u_{n}\left(\boldsymbol{k}_{\mathrm{TRIM}}\right)\left|\mathcal{T}^{T}\right| u_{n}\left(\boldsymbol{k}_{\mathrm{TRIM}}\right)\right\rangle\right\rangle \\
& =-\left\langle\left\langle u_{n}\left(\boldsymbol{k}_{\mathrm{TRIM}}\right)|\mathcal{T}| u_{n}\left(\boldsymbol{k}_{\mathrm{TRIM}}\right)\right\rangle\right\rangle,
\end{aligned}
$$

leading to

$$
\left\langle\left\langle u_{n}\left(\boldsymbol{k}_{\mathrm{TRIM}}\right)|\mathcal{T}| u_{n}\left(\boldsymbol{k}_{\mathrm{TRIM}}\right)\right\rangle\right\rangle=0 .
$$

This indicates that $\left|u_{n}\left(\boldsymbol{k}_{\mathrm{TRIM}}\right)\right\rangle$ and $\left.\mathcal{T}\left|u_{n}^{*}\left(\boldsymbol{k}_{\mathrm{TRIM}}\right)\right\rangle\right\rangle$ are biorthogonal [94] and linearly independent of each other. This Kramers degeneracy at time-reversal-invariant momenta is retained as long as reciprocity in Eq. (5) is respected.

Now, suppose the Chern number of $\eta H(\boldsymbol{k})$ is 1 . In the presence of Hermiticity, a pair of helical edge states appears and crosses at a time-reversal-invariant momentum. The bulk spectrum remains real because of pseudo-Hermiticity as long as the gap for the real part of the spectrum is open. On the other hand, the helical edge states are gapless and hence pseudo-Hermiticity alone cannot ensure their real spectrum. However, reciprocity and the consequent Kramers degeneracy ensure the real spectrum of the helical edge states. In fact, if the pair of the helical edge states mixed with each other and formed a complex-conjugate pair, Kramers degeneracy at the time-reversal-invariant momentum would be lifted, which is forbidden in the presence of reciprocity. Thus, the spectrum is entirely real for both bulk and edges as a consequence of the combination of pseudo-Hermiticity and reciprocity.

Next, suppose the Chern number of $\eta H(\boldsymbol{k})$ is 2 . In contrast to the previous case, two pairs of helical edge states appear, and neither of them necessarily crosses at time-reversalinvariant momenta. No degeneracy is guaranteed away from time-reversal-invariant momenta even in the presence of reciprocity. As a result, the helical edge states can mix with each other and form complex-conjugate pairs with exceptional points. Still, the bulk spectrum is real as long as the gap for the real part of the spectrum remains open. Thus, the system supports two pairs of helical lasing edge states. A model of such a symmetry-protected topological laser is provided in Refs. [31,56].

Notably, the bulk spectrum can change according to boundary conditions. This is a unique feature of non-Hermitian systems called the non-Hermitian skin effect $[35,44,46]$. However, when the bulk spectrum is real because of pseudoHermiticity (or parity-time symmetry), no skin effect occurs, i.e., the bulk spectrum under the periodic boundary conditions and that under the open boundary conditions always coincide with each other [31,56].

\section{Complex spectra in $\mathbb{Z}_{2}$ topological insulators}

Symmetry in Eqs. (1) and (2) for Hermitian Hamiltonians can be respectively generalized to non-Hermitian systems in a different manner as

$$
\begin{gathered}
\mathcal{T} H^{*}(\boldsymbol{k}) \mathcal{T}^{-1}=H(-\boldsymbol{k}), \quad \mathcal{T} \mathcal{T}^{*}=-1, \\
\eta H(\boldsymbol{k}) \eta^{-1}=H(\boldsymbol{k}), \quad \eta^{2}=1 .
\end{gathered}
$$

In the presence of Hermiticity, Eqs. (14) and (15), respectively, coincide with Eqs. (5) and (6), both of which reduce to Eqs. (1) and (2). However, this is not the case for nonHermitian Hamiltonians because of the distinction between complex conjugation and transposition [i.e., $H^{*}(\boldsymbol{k}) \neq H^{T}(\boldsymbol{k})$ ]. Whereas time-reversal symmetry in Eq. (14) leads to Kramers degeneracy for eigenstates with real eigenenergies [45], it results in no degeneracy for generic eigenstates with complex eigenenergies. This is contrasted with reciprocity in Eq. (5), which ensures Kramers degeneracy for all the eigenstates with complex eigenenergies. Furthermore, symmetry in Eq. (15) does not ensure the reality of the spectrum contrary to pseudoHermiticity in Eq. (6). Therefore, the other generalization in Eqs. (14) and (15) does not generally lead to the real spectra of non-Hermitian topological systems.

For example, a non-Hermitian extension of the BHZ model with Eq. (14) is investigated in the Appendix. Because of the symmetry protection, the topological phase and the helical edge states survive even in the presence of non-Hermiticity. However, non-Hermiticity mixes these helical edge states and creates a pair of exceptional points, and the Kramers degeneracy at the time-reversal-invariant momentum is lifted. Consequently, the edge spectrum generally becomes complex. In contrast to this extension, another non-Hermitian extension of the BHZ model with Eq. (5), which we consider in the subsequent sections, can possess entirely real spectra even in the presence of non-Hermiticity. 


\section{CONTINUUM DIRAC HAMILTONIAN}

Using non-Hermitian Dirac Hamiltonians with parity-time symmetry, Ref. [32] showed that entirely real spectra of both bulk and edges are impossible. As discussed above, however, the discussion there is not directly applicable in the presence of additional symmetry such as pseudo-Hermiticity and reciprocity. To confirm this fact, we consider a nonHermitian Dirac Hamiltonian and its spectrum in a similar manner to Ref. [32]. A non-Hermitian Dirac Hamiltonian having reciprocity in Eq. (5) and pseudo-Hermiticity in Eq. (6) is generally described by

$$
H(\boldsymbol{k})=\left(\sigma_{z}+i \gamma \sigma_{x}\right) k_{x} \tau_{x}+k_{y} \tau_{y}+\Delta \tau_{z} .
$$

Here, $\gamma \in \mathbb{R}$ describes the degree of non-Hermiticity, and $\Delta \in \mathbb{R}$ describes the mass parameter that determines the topological phases. This Dirac model indeed respects reciprocity in Eq. (5) and pseudo-Hermiticity in Eq. (6) (i.e., $\mathcal{T}=i \sigma_{y}$ and $\left.\eta=\sigma_{z}\right)$ :

$$
\begin{aligned}
\left(i \sigma_{y}\right) H^{T}(\boldsymbol{k})\left(i \sigma_{y}\right)^{-1} & =H(-\boldsymbol{k}), \quad\left(i \sigma_{y}\right)\left(i \sigma_{y}\right)^{*}=-1, \\
\sigma_{z} H^{\dagger}(\boldsymbol{k}) \sigma_{z}^{-1} & =H(\boldsymbol{k}), \quad \sigma_{z}^{2}=1 .
\end{aligned}
$$

The bulk spectrum is readily obtained as

$$
E(\boldsymbol{k})= \pm \sqrt{\left(1-\gamma^{2}\right) k_{x}^{2}+k_{y}^{2}+\Delta^{2}},
$$

which is entirely real for $|\gamma| \leqslant 1$ as a direct consequence of pseudo-Hermiticity in Eq. (18). It is twofold degenerate because of reciprocity in Eq. (17).

Even though the bulk spectrum is entirely real, the edge spectrum is not necessarily real. In fact, Ref. [32] showed that non-Hermiticity mixes a pair of edge states and makes the edge spectrum complex in a large class of non-Hermitian topological insulators. Still, the Dirac Hamiltonian in Eq. (16) possesses the entirely real spectrum even for the edges because of additional pseudo-Hermiticity and reciprocity. To see this, we consider an interface across which topological phases change. We assume that the system is uniform along the $x$ direction and has a domain wall at $y=0$. For the region $y>0(y<0)$, the mass parameter is assumed to be $\Delta(y)>0$ $[\Delta(y)<0]$. The corresponding continuum Hamiltonian reads

$$
H\left(k_{x}, y\right)=\left(\sigma_{z}+i \gamma \sigma_{x}\right) k_{x} \tau_{x}-i \tau_{y} \frac{\partial}{\partial y}+\Delta(y) \tau_{z} .
$$

For $k_{x}=0$, a Kramers pair of zero-energy bound states appears around the interface $y=0$. Solving the Shrödinger equation

$$
\left[-i \tau_{y} \frac{\partial}{\partial y}+\Delta(y) \tau_{z}\right]\left|\Psi_{\uparrow(\downarrow)}\right\rangle=0,
$$

we have

$$
\left|\Psi_{\uparrow(\downarrow)}\right\rangle=e^{-\int_{0}^{y} \Delta\left(y^{\prime}\right) d y^{\prime}}|\uparrow(\downarrow)\rangle|-\rangle,
$$

where $|\uparrow(\downarrow)\rangle$ and $|-\rangle$ are the eigenstates of $\sigma_{z}$ and $\tau_{x}$, respectively [i.e., $\sigma_{z}|\uparrow(\downarrow)\rangle=+(-)|\uparrow(\downarrow)\rangle$ and $\left.\tau_{x}|-\rangle=-|-\rangle\right]$. Away from the time-reversal-invariant momentum $k_{x}=0$, these boundary states have nonzero eigenenergies, which form the energy dispersion of the helical boundary states. The effective boundary Hamiltonian around $k_{x}=0$ is obtained as

$$
\begin{aligned}
H_{\text {edge }}\left(k_{x}, y\right) & \simeq\left(\begin{array}{cc}
\left\langle\Psi_{\uparrow}|H| \Psi_{\uparrow}\right\rangle & \left\langle\Psi_{\downarrow}|H| \Psi_{\uparrow}\right\rangle \\
\left\langle\Psi_{\uparrow}|H| \Psi_{\downarrow}\right\rangle & \left\langle\Psi_{\downarrow}|H| \Psi_{\downarrow}\right\rangle
\end{array}\right) \\
& =e^{-2 \int_{0}^{y} \Delta\left(y^{\prime}\right) d y^{\prime}}\left(\sigma_{z}+i \gamma \sigma_{x}\right) k_{x} .
\end{aligned}
$$

The energy dispersion is given as

$$
E_{\text {edge }}\left(k_{x}\right)= \pm \sqrt{1-\gamma^{2}} k_{x},
$$

which is indeed real for $|\gamma| \leqslant 1$.

We again stress that Kramers degeneracy plays a crucial role in the reality of the boundary spectrum. In the absence of reciprocity in Eq. (17), the Kramers degeneracy at $k_{x}=$ 0 is lifted by non-Hermitian perturbations and the boundary spectrum becomes complex, as discussed in Ref. [32]. In the presence of reciprocity, by contrast, the Kramers degeneracy cannot be lifted and the boundary spectrum remains real.

\section{NON-HERMITIAN BERNEVIG-HUGHES-ZHANG MODEL}

\section{A. Model and symmetry}

As a prime example of the preceding discussion, we consider a non-Hermitian extension of the BHZ model. The Hamiltonian in momentum space is given as

$$
\begin{aligned}
H_{\mathrm{BHZ}}(\boldsymbol{k})= & \left(m+t \cos k_{x}+t \cos k_{y}\right) \tau_{z}+t\left(\sin k_{y}\right) \tau_{y} \\
& +t\left(\sin k_{x}\right) \sigma_{z} \tau_{x}+i \gamma\left(\sin k_{x}\right) \sigma_{x} \tau_{x},
\end{aligned}
$$

where $t, m, \gamma \in \mathbb{R}$ are the hopping amplitude, the mass parameter, and the degree of non-Hermiticity, respectively. We assume $t, \gamma \geqslant 0$ without loss of generality. In the absence of non-Hermiticity (i.e., $\gamma=0$ ), Eq. (25) reduces to the original Hermitian BHZ model in Eq. (4).

Around the time-reversal-invariant momentum $\boldsymbol{k}=0$, the non-Hermitian BHZ model $H_{\mathrm{BHZ}}(\boldsymbol{k})$ reduces to the continuum Dirac model in Sec. III (i.e., $t=1$ and $\Delta=m+2 t$ ). It respects reciprocity in Eq. (17) and pseudo-Hermiticity in Eq. (18). In addition, it respects parity (spatial-inversion) symmetry:

$$
\tau_{z} H(\boldsymbol{k}) \tau_{z}^{-1}=H(-\boldsymbol{k}), \quad \tau_{z}^{2}=1 .
$$

As a combination of these symmetry, $H_{\mathrm{BHZ}}(\boldsymbol{k})$ also respects parity-time symmetry:

$$
\left(\tau_{z} \sigma_{x}\right) H^{*}(\boldsymbol{k})\left(\tau_{z} \sigma_{x}\right)^{-1}=H(\boldsymbol{k}), \quad\left(\tau_{z} \sigma_{x}\right)^{2}=1 .
$$

While reciprocity and pseudo-Hermiticity are internal symmetry, parity symmetry and parity-time symmetry are spatial symmetry, the latter of which is fragile against disorder.

\section{B. Phase diagram}

The spectrum of $H_{\mathrm{BHZ}}(\boldsymbol{k})$ is obtained as

$$
\begin{aligned}
E(\boldsymbol{k})= & \pm\left[\left(m+t \cos k_{x}+t \cos k_{y}\right)^{2}\right. \\
& \left.+\left(t^{2}-\gamma^{2}\right) \sin ^{2} k_{x}+t^{2} \sin ^{2} k_{y}\right]^{1 / 2} .
\end{aligned}
$$

A topological phase persists as long as a gap for the real part of eigenenergies is open [i.e., $\forall \boldsymbol{k} \operatorname{Re} E(\boldsymbol{k}) \neq 0$ ]; vanishing of the real part of eigenenergies [i.e., $\exists \boldsymbol{k} \operatorname{Re} E(\boldsymbol{k})=$ 
0] can be considered to be a topological phase transition. Here, $E(\boldsymbol{k})$ in Eq. (28) is either real or purely imaginary. In particular, $E(\boldsymbol{k})$ is always real for the time-reversal-invariant momenta $\boldsymbol{k}_{\mathrm{TRIM}} \in\{(0,0),(0, \pi),(\pi, 0),(\pi, \pi)\}$. Thus, if an energy gap for the real part of the spectrum is closed, it holds $E(\boldsymbol{k})=0$ for some $\boldsymbol{k}$, and vice versa. This reduces to the following gapless conditions according to $t$ and $\gamma$.

(i) $\gamma<t$. Since we have

$$
\begin{aligned}
& \left(m+t \cos k_{x}+t \cos k_{y}\right)^{2} \geqslant 0, \\
& \left(t^{2}-\gamma^{2}\right) \sin ^{2} k_{x} \geqslant 0, \quad t^{2} \sin ^{2} k_{y} \geqslant 0,
\end{aligned}
$$

$E(\boldsymbol{k})=0$ leads to

$$
m+t \cos k_{x}+t \cos k_{y}=\sin k_{x}=\sin k_{y}=0 .
$$

Hence, we have

$$
\begin{array}{lll}
m=-2 t & \text { for } & \boldsymbol{k}_{0}=(0,0), \\
m=0 & \text { for } & \boldsymbol{k}_{0}=(0, \pi),(\pi, 0), \\
m=2 t & \text { for } & \boldsymbol{k}_{0}=(\pi, \pi),
\end{array}
$$

where $\boldsymbol{k}_{0}$ is a momentum satisfying $E\left(\boldsymbol{k}_{0}\right)=0$.

(ii) $\gamma=t$. Since $E(\boldsymbol{k})=0$ leads to

$$
m+t \cos k_{x}+t \cos k_{y}=\sin k_{y}=0,
$$

we have

$$
\begin{array}{ll}
-2 t \leqslant m \leqslant 0 & \text { for } \quad \boldsymbol{k}_{0}=(\arccos (1+m / t), 0), \\
0 \leqslant m \leqslant 2 t & \text { for } \quad \boldsymbol{k}_{0}=(\arccos (1-m / t), \pi) .
\end{array}
$$

(iii) $\gamma>t$. Since we have

$$
\begin{aligned}
& E^{2}(0,0)=(m+2 t)^{2} \geqslant 0, \\
& E^{2}(0, \pi)=E^{2}(\pi, 0)=m^{2} \geqslant 0, \\
& E^{2}(\pi, \pi)=(m-2 t)^{2} \geqslant 0,
\end{aligned}
$$

there exists $\boldsymbol{k}_{0}$ satisfying $E\left(\boldsymbol{k}_{0}\right)=0$ if and only if the minimum of $E^{2}(\boldsymbol{k})$ is nonpositive. Then, we have

$$
\begin{aligned}
E^{2}(\boldsymbol{k})= & 2 t\left(m+t \cos k_{x}\right) \cos k_{y} \\
& +\left(m+t \cos k_{x}\right)^{2}+\left(t^{2}-\gamma^{2}\right) \sin ^{2} k_{x}+t^{2},
\end{aligned}
$$

which implies that $E^{2}(\boldsymbol{k})$ is minimum for $k_{y}=0$ or $k_{y}=\pi$. Now, $E^{2}\left(k_{x}, 0\right)$ is given as

$$
\begin{aligned}
E^{2}\left(k_{x}, 0\right)= & \gamma^{2}\left[\cos k_{x}+\frac{t(m+t)}{\gamma^{2}}\right]^{2} \\
& +\left(1-\frac{t^{2}}{\gamma^{2}}\right)\left[(m+t)^{2}-\gamma^{2}\right],
\end{aligned}
$$

and $E^{2}\left(k_{x}, 0\right)$ is nonnegative for $k_{x}=0$ and $k_{x}=\pi$. Thus, we have $E\left(\boldsymbol{k}_{0}\right)=0$ for

$$
\boldsymbol{k}_{0}=\left(\arccos \left(-\frac{t(m+t)}{\gamma^{2}}\right), 0\right)
$$

if and only if

$$
\left|\frac{t(m+t)}{\gamma^{2}}\right| \leqslant 1, \quad(m+t)^{2}-\gamma^{2}<0
$$

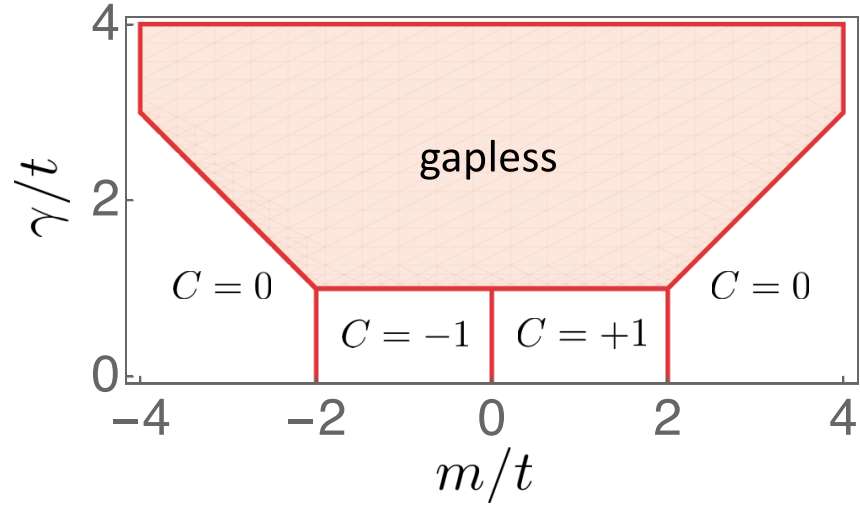

FIG. 1. Phase diagram of the non-Hermitian Bernevig-HughesZhang model. Topological phase transitions occur at the phase boundaries, at which an energy gap for the real part of the complex spectrum closes. Each gapped phase is characterized by the Chern number $C \in \mathbb{Z}$ of $\eta H(\boldsymbol{k})$. A pair of helical edge states appears for $|C|=1$, whereas no edge states appear for $C=0$.

are satisfied; these inequalities reduce to $\gamma>|m+t|$. Similarly, we have $E\left(\boldsymbol{k}_{0}\right)=0$ for

$$
\boldsymbol{k}_{0}=\left(\arccos \left(-\frac{t(m-t)}{\gamma^{2}}\right), \pi\right)
$$

as long as $\gamma>|m-t|$ is satisfied.

The obtained phase diagram is provided in Fig. 1. Since topology is invariant unless an energy gap is closed, the topological invariant in each gapped phase is obtained by continuously deforming the non-Hermitian system into the corresponding Hermitian system without closing the energy gap. In the absence of non-Hermiticity (i.e., $\gamma=0$ ), we have

$$
\begin{aligned}
\eta H_{\mathrm{BHZ}}(\boldsymbol{k})= & \left(m+t \cos k_{x}+t \cos k_{y}\right) \tau_{z} \sigma_{z} \\
& +t\left(\sin k_{y}\right) \tau_{y} \sigma_{z}+t\left(\sin k_{x}\right) \tau_{x} .
\end{aligned}
$$

The Chern number $C$ of $\eta H_{\mathrm{BHZ}}(\boldsymbol{k})$ with $\gamma=0$ is readily obtained as

$$
C=\left\{\begin{array}{ccc}
\operatorname{sgn}(m) & \text { for } & |m / t|<2 \\
0 & \text { for } & |m / t|>2 .
\end{array}\right.
$$

This Chern number $C$ is the topological invariant of $H_{\mathrm{BHZ}}(\boldsymbol{k})$ in the gapped phases, as shown in Fig. 1.

\section{Helical edge states}

Corresponding to the nontrivial topology of the bulk, a pair of helical edge states appears under the open boundary conditions. We here investigate the non-Hermitian $\mathrm{BHZ}$ model with periodic boundaries in the $x$ direction and open boundaries in the $y$ direction,

$$
\begin{aligned}
\hat{H}_{\mathrm{BHZ}}= & \sum_{k_{x}, y}\left\{\left[\hat{c}_{k_{x}, y+1}^{\dagger} \frac{t\left(\tau_{z}+i \tau_{y}\right)}{2} \hat{c}_{k_{x}, y}+\text { H.c. }\right]\right. \\
& +\hat{c}_{k_{x}, y}^{\dagger}\left[\left(m+t \cos k_{x}\right) \tau_{z}+t\left(\sin k_{x}\right) \sigma_{z} \tau_{x}\right. \\
& \left.\left.+i \gamma\left(\sin k_{x}\right) \sigma_{x} \tau_{x}\right] \hat{c}_{k_{x}, y}\right\}
\end{aligned}
$$




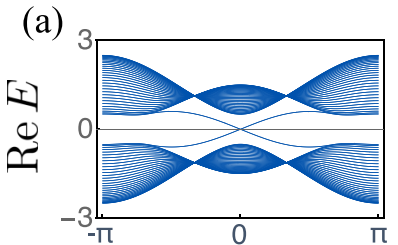

(b)

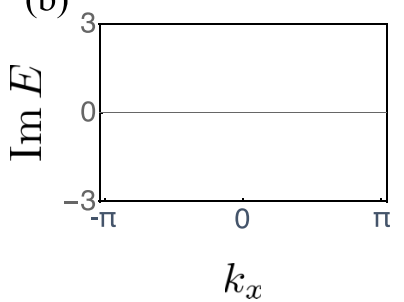

(c)

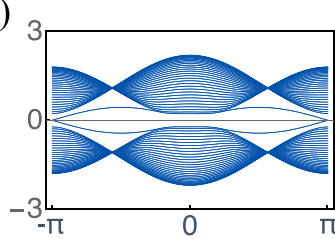

(d)

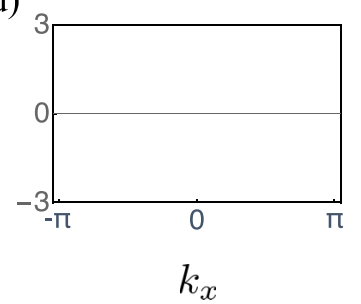

(e)

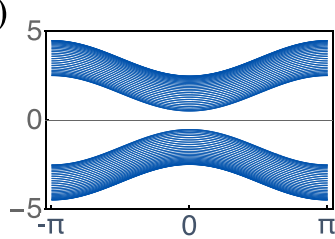

(f)

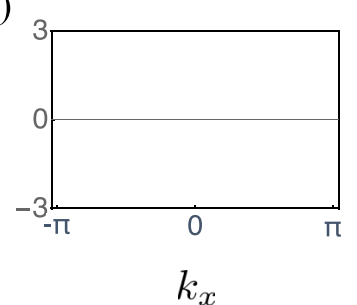

(g)

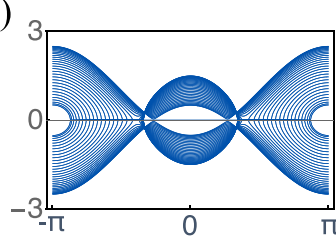

(h)

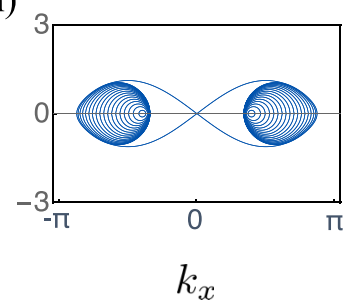

FIG. 2. Complex spectrum of the non-Hermitian Bernevig-Hughes-Zhang model. The open boundary conditions are imposed in the $y$ direction (30 sites), whereas the periodic boundary conditions are imposed in the $x$ direction, along which the wave number $k_{x}$ is defined. (a, b) Gapped and topologically nontrivial phase $(t=1.0, m=-0.5, \gamma=0.8 ; C=-1)$. A pair of helical edge states appears around $k_{x}=0$. (c, d) Gapped and topologically nontrivial phase $(t=1.0, m=0.2, \gamma=0.9 ; C=+1)$. A pair of helical edge states appears around $k_{x}= \pm \pi$. (e, f) Gapped and topologically trivial phase $(t=1.0, m=-2.5, \gamma=1.0 ; C=0)$. No edge states appear between the gapped bands. (g, h) Gapless phase $(t=1.0, m=-0.5, \gamma=1.5)$. The spectrum is entirely real in the gapped phases $(\mathrm{a}-\mathrm{f})$, but it is complex in the gapless phase $(\mathrm{g}, \mathrm{h})$.

where $\hat{c}_{k_{x}, y}\left(\hat{c}_{k_{x}, y}^{\dagger}\right)$ annihilates (creates) a particle at site $y$ with momentum $k_{x}$ that has 4 internal degrees of freedom. The spectrum is shown in Fig. 2. In the gapped phases with nontrivial topology, a pair of helical edge states indeed appears at both edges [Figs. 2(a)-2(d)]. On the other hand, no edge states appear in the gapped phase with trivial topology [Figs. 2(e) and 2(f)]. The spectra are entirely real even in the presence of the edge states. When non-Hermiticity is sufficiently strong and the gap for the real part of the spectrum closes, the bulk spontaneously breaks pseudo-Hermiticity and its spectrum becomes complex [Figs. 2(g) and 2(h)].

We note that no skin effects occur in $H_{\mathrm{BHZ}}(\boldsymbol{k})$. Thus, similar results are obtained under different types of the open boundary conditions, i.e., the open boundary conditions in the $x$ direction and the periodic boundary conditions in the $y$ direction, or the open boundary conditions in both $x$ and $y$ directions. This is contrasted with non-Hermitian systems that exhibit skin effects, including non-Hermitian Chern insulators $[44,47]$.

The energy dispersions and wave functions of the helical edge states are analytically obtained in the following manner. Let us consider a pair of helical edge states localized around $y=1$. The edge states are denoted as

$$
\hat{\Psi}_{\text {edge }} \propto \sum_{y} \lambda^{y-1}\left(\hat{c}_{k_{x}, y}^{\dagger} \vec{v}\right),
$$

where $\lambda$ is a parameter that determines the localization length [given by $-(\log |\lambda|)^{-1}$ ], and $\vec{v}$ is a four-component vector that describes the internal degrees of freedom. Then, the Schrödinger equation $\left[\hat{H}, \hat{\Psi}_{\text {edge }}\right]=E_{\text {edge }} \hat{\Psi}_{\text {edge }}$ reduces to

$$
\left(\lambda^{-1} T+M+\lambda T^{\dagger}\right) \vec{v}=E_{\text {edge }} \vec{v}
$$

in the bulk and

$$
\left(M+\lambda T^{\dagger}\right) \vec{v}=E_{\text {edge }} \vec{v}
$$

at the edge. Here, $T$ and $M$ are defined as

$$
\begin{aligned}
T & :=\frac{t\left(\tau_{z}+i \tau_{y}\right)}{2}, \\
M & :=\left(m+t \cos k_{x}\right) \tau_{z}+t\left(\sin k_{x}\right) \tau_{x} \sigma_{z}+i \gamma\left(\sin k_{x}\right) \tau_{x} \sigma_{x} .
\end{aligned}
$$

In addition, we take the semi-infinite limit and neglect the effect of the other edge. Equations (44) and (45) lead to $T \vec{v}=0$, which implies

$$
\vec{v}=\left(\begin{array}{c}
\vec{v}_{\sigma} \\
-\vec{v}_{\sigma}
\end{array}\right),
$$

with a two-component vector $\vec{v}_{\sigma}$ that acts in the space of $\sigma_{i}$ 's. Using Eq. (44) or Eq. (45), we have

$$
\begin{gathered}
\left(\lambda t+m+t \cos k_{x}\right) \vec{v}_{\sigma}=0, \\
{\left[t\left(\sin k_{x}\right) \sigma_{z}+i \gamma\left(\sin k_{x}\right) \sigma_{x}\right] \vec{v}_{\sigma}=-E_{\text {edge }} \vec{v}_{\sigma} .}
\end{gathered}
$$

Since $\vec{v}_{\sigma}$ is nonvanishing, Eq. (48) leads to

$$
\lambda=-\frac{m}{t}-\cos k_{x}
$$

which determines the localization length of the helical edge states. Here, $\lambda$ should be less than 1 so that the edge states can be normalized. This gives $\left|m / t+\cos k_{x}\right|<1$. For the presence of the helical edge states, there exists a wave number $k_{x}$ that satisfies this inequality, which then leads to $|m / t|<2$. This condition is compatible with the phase diagram in Fig. 1. Furthermore, Eq. (49) implies that $\vec{v}_{\sigma}$ is an eigenstate of the $2 \times 2$ matrix $t\left(\sin k_{x}\right) \sigma_{z}+i \gamma\left(\sin k_{x}\right) \sigma_{x}$ with the eigenenergy $-E_{\text {edge }}$, which gives

$$
E_{\text {edge }}\left(k_{x}\right)= \pm \sqrt{t^{2}-\gamma^{2}} \sin k_{x} .
$$

Thus, the spectrum of the helical edge states is indeed real for $\gamma<t$. The obtained analytical results are consistent with 

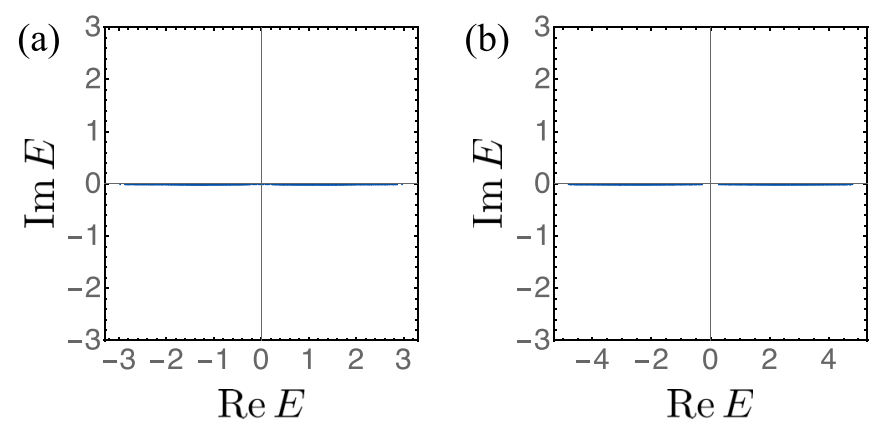

FIG. 3. Complex spectrum of the non-Hermitian BernevigHughes-Zhang model with disorder. The open boundary conditions are imposed in both $x$ and $y$ directions $(30 \times 30$ sites $)$. Even in the presence of disorder, the spectrum is entirely real for both (a) topological phase $\left(t=1.0, m_{x, y}=-0.5+2.0 \epsilon_{x, y}, \gamma=0.8\right)$ and (b) trivial phase $\left(t=1.0, m_{x, y}=-2.5+2.0 \epsilon_{x, y}, \gamma=1.0\right)$. Here, $\epsilon_{x, y}$ is a random variable uniformly distributed over $[-0.5,0.5]$.

the numerical results in Fig. 2, as well as the results for the continuum Dirac Hamiltonian in Sec. III.

\section{Robustness to disorder}

The entirely real spectra in the non-Hermitian BHZ model are robust to disorder. To see this, we investigate the following disordered model:

$$
\begin{aligned}
\hat{H}_{\mathrm{BHZ}}= & \sum_{x, y}\left\{\left[\hat{c}_{x, y+1}^{\dagger} \frac{t\left(\tau_{z}+i \tau_{y}\right)}{2} \hat{c}_{x, y}+\text { H.c. }\right]\right. \\
& +\left[\hat{c}_{x+1, y}^{\dagger} \frac{t\left(\tau_{z}+i \sigma_{z} \tau_{x}\right)-\gamma \sigma_{x} \tau_{x}}{2} \hat{c}_{x, y}\right. \\
& \left.+\hat{c}_{x, y}^{\dagger} \frac{t\left(\tau_{z}-i \sigma_{z} \tau_{x}\right)+\gamma \sigma_{x} \tau_{x}}{2} \hat{c}_{x+1, y}\right] \\
& \left.+\hat{c}_{x, y}^{\dagger}\left(m_{x, y} \tau_{z}\right) \hat{c}_{x, y}\right\},
\end{aligned}
$$

where the open boundary conditions are imposed in both $x$ and $y$ directions. In contrast to the clean model, the mass parameters $m_{x, y}$ depend on the lattice sites $x$ and $y$. As shown in Fig. 3, the spectrum of this disordered model is entirely real even in the presence of disorder. There, $m_{x, y}$ 's are uniformly distributed random variables. Such disorder breaks parity symmetry in Eq. (26) and parity-time symmetry in Eq. (27). On the other hand, reciprocity and pseudo-Hermiticity remain to be respected since they are internal symmetry.

In a similar manner to the clean model discussed in Sec. II B, the reality of the bulk spectrum is due to pseudoHermiticity. However, the discussion in Sec. II B is not directly applicable to the reality of the edge spectrum since it relies on translation invariance. Still, the real edge spectrum can be partially understood on the basis of the continuum models in Sec. III. Suppose the system includes disorder solely along the $y$ direction, and translation invariance is respected along the $x$ direction. Then, the space-dependent mass parameter $\Delta(y)$ of the continuum model in Eq. (20), which corresponds to $m_{x, y}$ of the lattice model in Eq. (52), only changes the eigenstates and has no effect on the spectrum, as shown in Eq. (24).

It is also notable that disorder generally tends to give rise to real spectra and stabilize non-Hermitian systems. Prime examples include the Hatano-Nelson model [26]. It is a time-reversal-invariant system in one dimension whose hopping amplitudes exhibit asymmetry as the degree of non-Hermiticity. Because of this non-Hermiticity, it possesses a complex spectrum in the absence of disorder. In the presence of disorder, by contrast, some eigenstates are localized and uncorrelated with other eigenstates. Consequently, these localized eigenstates have real eigenenergies. This disorder-induced real spectrum is stable against manybody interactions [18]. Thus, it is intuitively expected that disorder leads to the real spectra also in the non-Hermitian BHZ model, although symmetry or topology may change this behavior even qualitatively.

\section{POWER OSCILLATION}

Even when a non-Hermitian system possesses an entirely real spectrum, it exhibits unique phenomena that have no analogs in Hermitian systems. Eigenstates of a non-Hermitian Hamiltonian are biorthogonal to each other [94],

$$
\left\langle\left\langle u_{m} \mid u_{n}\right\rangle \propto \delta_{m n}, \quad\left\langle u_{m} \mid u_{n}\right\rangle\right\rangle \propto \delta_{m n},
$$

where $\left.\left|u_{n}\right\rangle\left(\left|u_{n}\right\rangle\right\rangle\right)$ is a right (left) eigenstate of the nonHermitian Hamiltonian $H$. Nevertheless, they are, in general, nonorthogonal to each other:

$$
\left\langle u_{m} \mid u_{n}\right\rangle \neq \delta_{m n}, \quad\left\langle\left\langle u_{m} \mid u_{n}\right\rangle\right\rangle \neq \delta_{m n} .
$$

An immediate physical consequence of the nonorthogonality between eigenstates is power oscillation. This is the oscillation of the norm (power) unique to non-Hermitian systems. When a wave function is initially prepared to be

$$
|\psi(0)\rangle=\sum_{n} c_{n}\left|u_{n}\right\rangle, \quad c_{n}:=\frac{\left\langle u_{n} \mid \psi(0)\right\rangle}{\left\langle\left\langle u_{n} \mid u_{n}\right\rangle\right.},
$$

it evolves into

$$
|\psi(t)\rangle=e^{-i H t}|\psi(0)\rangle=\sum_{n} c_{n} e^{-i E_{n} t}\left|u_{n}\right\rangle,
$$

where $E_{n}$ is the eigenenergy that corresponds to $\left|u_{n}\right\rangle$ and $\left.\left|u_{n}\right\rangle\right\rangle$. Its norm is given by

$$
\langle\psi(t) \mid \psi(t)\rangle=\sum_{m, n} c_{m}^{*} c_{n} e^{i\left(E_{m}^{*}-E_{n}\right) t}\left\langle u_{m} \mid u_{n}\right\rangle .
$$

In Hermitian systems, this reduces to

$$
\langle\psi(t) \mid \psi(t)\rangle=\sum_{n}\left|c_{n}\right|^{2}\left\langle u_{n} \mid u_{n}\right\rangle=\langle\psi(0) \mid \psi(0)\rangle
$$

because of the orthogonality between eigenstates (i.e., $\left\langle u_{m} \mid u_{n}\right\rangle \propto \delta_{m n}$ ) and the reality of eigenenergies (i.e., $E_{n}^{*}=$ $\left.E_{n}\right)$. In non-Hermitian systems, by contrast, eigenstates are in general nonorthogonal, and hence the norm $\langle\psi(t) \mid \psi(t)\rangle$ depends on time, which is a clear manifestation of nonunitarity of the dynamics resulting from coupling to an external environment. Notably, even when eigenenergies are entirely real, eigenstates are still nonorthogonal and the norm oscillates in contrast to unitary dynamics of Hermitian systems. 
This power oscillation was experimentally observed in the bulk of an open photonic lattice with balanced gain and loss [8]. A quantum counterpart arises as oscillation of quantum information flow between a system and its environment [17], which was observed in dissipative single photons [19]. Furthermore, we note in passing that the power oscillation has an analogy with the norm leakage in open chaotic systems [95].

The helical edge states oscillate in the non-Hermitian BHZ model. As an illustration, we investigate the non-Hermitian BHZ model $H_{\mathrm{BHZ}}$ with periodic boundaries in the $x$ direction and open boundaries in the $y$ direction, in a similar manner to Sec. IV C. The number of sites is $L_{x} \times L_{y}$. An eigenenergy and the corresponding right (left) eigenstate of $H_{\mathrm{BHZ}}\left(k_{x}\right)$ are, respectively, denoted as $E_{n}\left(k_{x}\right)$ and $\left.\left|u_{n}\left(k_{x}\right)\right\rangle\left(\left|u_{n}\left(k_{x}\right)\right\rangle\right\rangle\right)$ with $n=1,2, \ldots, 4 L_{y}$, where $H_{\mathrm{BHZ}}\left(k_{x}\right)$ is a Fourier transform of the original Hamiltonian $H_{\mathrm{BHZ}}$ along the $x$ direction. The eigenstates are normalized by

$$
\left\langle\left\langle u_{m}\left(k_{x}\right) \mid u_{n}\left(k_{x}^{\prime}\right)\right\rangle=\left\langle u_{m}\left(k_{x}\right) \mid u_{n}\left(k_{x}^{\prime}\right)\right\rangle\right\rangle=\delta_{m, n} \delta_{k_{x}, k_{x}^{\prime}} .
$$

Then a right (left) eigenstate of $H_{\mathrm{BHZ}}$ is given by $\left|k_{x}\right\rangle\left|u_{n}\left(k_{x}\right)\right\rangle$ $\left.\left(\left|k_{x}\right\rangle\left|u_{n}\left(k_{x}\right)\right\rangle\right\rangle\right)$ with

$\left|k_{x}\right\rangle:=\frac{1}{\sqrt{L_{x}}} \sum_{x=1}^{L_{x}} e^{i x k_{x}}|x\rangle, \quad k_{x} \in\left\{0, \frac{2 \pi}{L_{x}}, \ldots, \frac{2\left(L_{x}-1\right) \pi}{L_{x}}\right\}$.

Using these eigenstates, we expand the initial state $|\psi(0)\rangle:=$ $\sum_{x, y} c_{x y}|x\rangle|y\rangle$ as

$$
|\psi(0)\rangle=\sum_{k_{x}, n} c_{n}\left(k_{x}\right)\left|k_{x}\right\rangle\left|u_{n}\left(k_{x}\right)\right\rangle
$$

with

$$
c_{n}\left(k_{x}\right):=\frac{1}{\sqrt{L_{x}}} \sum_{x, y} c_{x y} e^{-i k_{x} x}\left\langle\left\langle u_{n}\left(k_{x}\right) \mid y\right\rangle .\right.
$$

This state evolves into

$$
\begin{aligned}
|\psi(t)\rangle & =e^{-i H_{\mathrm{BHZ}} t}|\psi(0)\rangle \\
& =\sum_{k_{x}, n} c_{n}\left(k_{x}\right) e^{-i E_{n}\left(k_{x}\right) t}\left|k_{x}\right\rangle\left|u_{n}\left(k_{x}\right)\right\rangle,
\end{aligned}
$$

and its amplitude at $y=y_{0}$ is

$$
\left|\left\langle y_{0} \mid \psi(t)\right\rangle\right|^{2}=\sum_{k_{x}}\left|\sum_{n} c_{n}\left(k_{x}\right)\left\langle y_{0} \mid u_{n}\left(k_{x}\right)\right\rangle e^{-i E_{n}\left(k_{x}\right) t}\right|^{2} .
$$

Figure 4 shows the evolutions of the population at the edge $y_{0}=1$ for each phase. There, an initial state is prepared to be a localized state at the edge $y_{0}=1$. In the topological phase, the wave packet remains localized because of the presence of the helical edge states, while some of the population is absorbed into the bulk. The helical edge states indeed exhibit oscillatory dynamics. Although the edge amplitude oscillates even in the Hermitian case, the oscillation is enhanced by non-Hermiticity and the consequent nonorthogonality. In the trivial phase, on the other hand, the wave packet quickly diffuses into the bulk since no edge states appear, which results in the monotonic decrease in the edge amplitudes in both Hermitian and non-Hermitian cases. Such power oscillation of the nonorthogonal edge states can in principle occur even

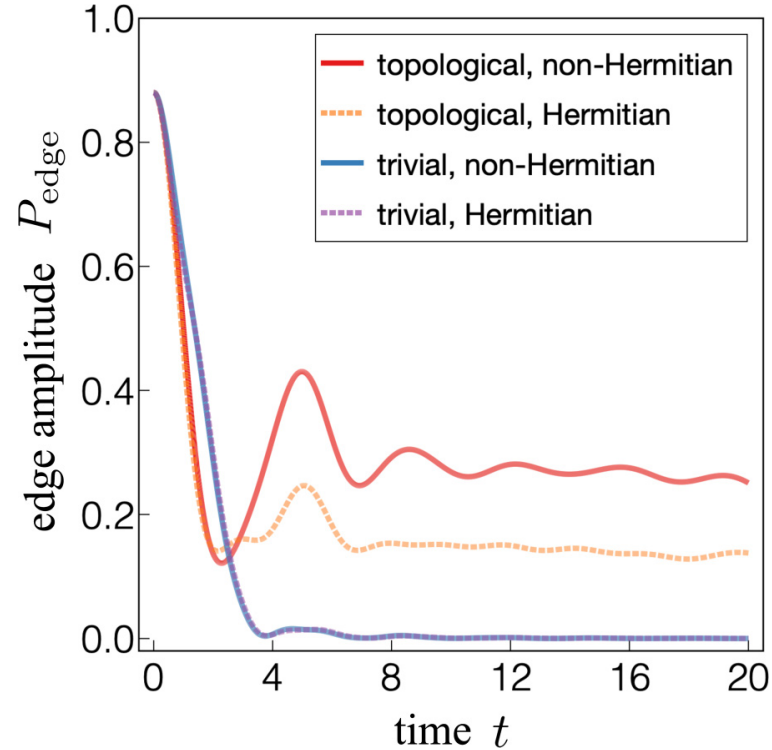

FIG. 4. Power oscillation at an edge in the non-Hermitian Bernevig-Hughes-Zhang model. An initial state is prepared to be a localized wave function $|\psi(0)\rangle \propto \sum_{x, y} e^{-(x-1)^{2} / 36-(y-1)^{2}}|x\rangle|y\rangle$, and the evolutions of the amplitude at the edge [i.e., $P_{\text {edge }}(t):=$ $\left.\left|\left\langle y=1\left|e^{-i H_{\mathrm{BHZ}} t}\right| \psi(0)\right\rangle\right|^{2}\right]$ are shown. The two-dimensional system consists of $30 \times 30$ sites and has periodic boundaries in the $x$ direction and open boundaries in the $y$ direction. The solid red curve shows the dynamics for the non-Hermitian topological phase $(t=1.0, m=$ $-0.5, \gamma=0.8)$, whereas the solid blue curve shows the dynamics for the non-Hermitian trivial phase $(t=1.0, m=-2.5, \gamma=1.0)$; the dotted orange curve shows the dynamics for the Hermitian topological phase $(t=1.0, m=-0.5, \gamma=0)$, whereas the dotted violet curve shows the dynamics for the Hermitian trivial phase $(t=1.0$, $m=-2.5, \gamma=0)$.

in non-Hermitian topological systems with complex spectra. However, it is in practice difficult to observe because amplification or attenuation dominates the nonunitary dynamics and clears away a signature of the power oscillation.

\section{DISCUSSION}

The reality of spectra is relevant to the stability of non-Hermitian systems. Nevertheless, non-Hermiticity often makes spectra of bulk or edges in topological insulators complex. In this work, we have shown that a combination of pseudo-Hermiticity and reciprocity (a variant of time-reversal symmetry) enables entirely real spectra even in non-Hermitian topological insulators. Thanks to pseudo-Hermiticity, the bulk spectra remain real as long as an energy gap for the real part of the spectrum is open. Still, the gapless edge states are not necessarily real solely in the presence of pseudoHermiticity. Instead, the reality of the edge spectrum is ensured by Kramers degeneracy due to reciprocity. As a prototypical example, we have illustrated this with a non-Hermitian extension of the BHZ model [86]. Although Ref. [32] showed that entirely real spectra of both bulk and edges are impossible in a large class of non-Hermitian topological insulators with parity-time symmetry, the discussion there is not directly 
applicable in the presence of additional symmetry such as pseudo-Hermiticity and reciprocity.

Non-Hermitian topological insulators with real spectra can be experimentally realized in various synthetic materials. In fact, Hermitian $\mathbb{Z}_{2}$ topological insulators including the $\mathrm{BHZ}$ model can be created in a variety of classical systems, such as photonic systems [96,97], mechanical metamaterials [98], and electric circuits [99]. In these systems, non-Hermiticity such as gain or loss, as well as asymmetric hopping, can be introduced by judiciously controlling the external coupling to the environment [1,2]. An experimental signature of the entirely real spectra is the power oscillation of helical edge states, which is induced by the nonorthogonality due to nonHermiticity.

Moreover, real spectra may be feasible in non-Hermitian topological insulators with different symmetry in different spatial dimensions. As long as internal symmetry is relevant, they can be systematically explored on the basis of the topological classification of non-Hermitian systems [56]. Spatial symmetry can also enrich band structures of non-Hermitian systems. Furthermore, a recent work demonstrated the entirely real spectrum in a non-Hermitian topological quasicrystal in one dimension [68]. Further research is warranted for such new types of non-Hermitian topological insulators with real spectra.

\section{ACKNOWLEDGMENTS}

We thank Dmitry V. Savin and Qi-Bo Zeng for bringing Ref. [95] and Refs. [36] and [68], respectively, to our attention. This work was supported by a Grant-in-Aid for Scientific Research on Innovative Areas "Topological Materials Science" (KAKENHI Grant No. JP15H05855) from the Japan Society for the Promotion of Science (JSPS), and JST CREST Grant No. JPMJCR19T2. K.K. was supported by KAKENHI Grant No. JP19J21927 from the JSPS. M.S. was supported by KAKENHI Grants No. JP17H02922 and No. JP20H00131 from the JSPS.

\section{APPENDIX: NON-HERMITIAN BERNEVIG-HUGHES-ZHANG MODEL PROTECTED BY TIME-REVERSAL SYMMETRY}

In Sec. IV, we have investigated a non-Hermitian extension of the $\mathrm{BHZ}$ model protected by reciprocity. While reciprocity is equivalent to time-reversal symmetry in Hermitian systems, this is not the case in non-Hermitian systems. In fact, time-reversal symmetry in non-Hermitian spinful systems is defined by Eq. (14), which is different from reciprocity in Eq. (5). Both symmetry can protect the topological phase of the BHZ model as long as the real part of the spectrum is gapped. However, the real spectrum of the helical edge states cannot be protected by time-reversal symmetry, which contrasts with reciprocity.

To see the difference between reciprocity and time-reversal symmetry, we here consider another non-Hermitian extension
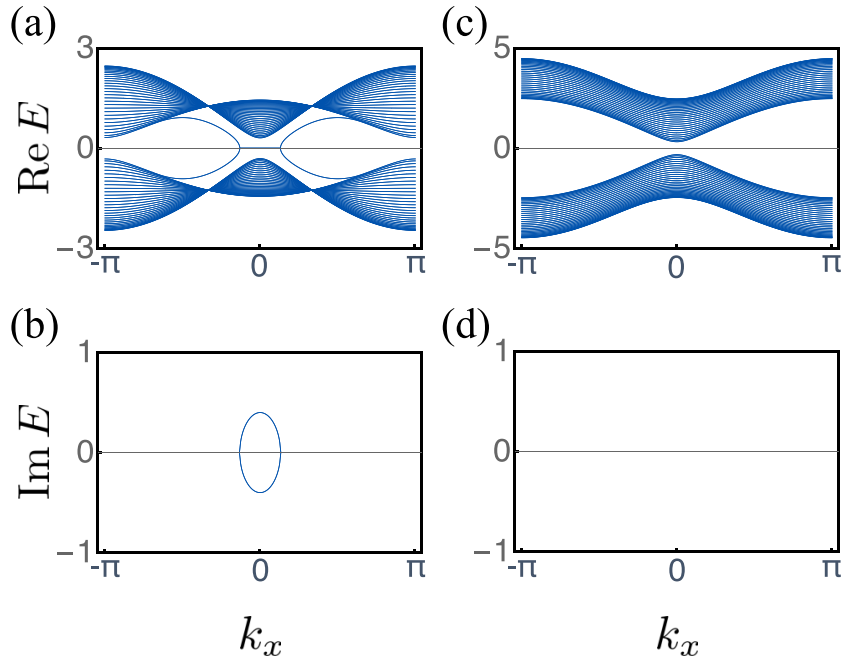

(d)

FIG. 5. Complex spectrum of the non-Hermitian BernevigHughes-Zhang model protected by time-reversal symmetry. The open boundary conditions are imposed in the $y$ direction (30 sites), whereas the periodic boundary conditions are imposed in the $x$ direction, along which the wave number $k_{x}$ is defined. (a, b) Gapped and topologically nontrivial phase $(t=1.0, m=-0.5, \gamma=0.4)$. A pair of helical edge states appears around $k_{x}=0$. The helical edge states coalesce with each other and form exceptional points, leading to the complex spectrum at the edges. (c, d) Gapped and topologically trivial phase $(t=1.0, m=-2.5, \gamma=0.4)$. No edge states appear between the gapped bands, and the spectrum is entirely real.

of the BHZ model protected by time-reversal symmetry:

$$
\begin{aligned}
\tilde{H}_{\mathrm{BHZ}}(\boldsymbol{k})= & \left(m+t \cos k_{x}+t \cos k_{y}\right) \tau_{z}+t\left(\sin k_{y}\right) \tau_{y} \\
& +t\left(\sin k_{x}\right) \sigma_{z} \tau_{x}+i \gamma \sigma_{x} \tau_{x} .
\end{aligned}
$$

In a similar manner to the previous model $H_{\mathrm{BHZ}}(\boldsymbol{k})$, this model $\tilde{H}_{\mathrm{BHZ}}(\boldsymbol{k})$ respects pseudo-Hermiticity in Eq. (6) with $\eta=\sigma_{z}$ :

$$
\sigma_{z} \tilde{H}_{\mathrm{BHZ}}^{\dagger}(\boldsymbol{k}) \sigma_{z}=\tilde{H}_{\mathrm{BHZ}}(\boldsymbol{k}) \text {. }
$$

By contrast, it does not respect reciprocity in Eq. (5); instead, it respects time-reversal symmetry in Eq. (14) with $\mathcal{T}=i \sigma_{y}$ :

$$
\left(i \sigma_{y}\right) \tilde{H}_{\mathrm{BHZ}}^{*}(\boldsymbol{k})\left(i \sigma_{y}\right)^{-1}=\tilde{H}_{\mathrm{BHZ}}(-\boldsymbol{k}) .
$$

Notably, a similar non-Hermitian quantum spin Hall insulator was also investigated in Ref. [45].

The spectrum of $\tilde{H}_{\mathrm{BHZ}}(\boldsymbol{k})$ is shown in Fig. 5. The bulk spectrum is real as long as the bulk bands are gapped, which is due to pseudo-Hermiticity. Between the gapped bulk bands, a pair of helical edge states appears in the topological phase. In the previous model $H_{\mathrm{BHZ}}(\boldsymbol{k})$, these helical edge states are forbidden to mix with each other because of the Kramers degeneracy. However, time-reversal symmetry does not impose such a constraint in non-Hermitian systems. Consequently, the helical edge states coalesce with each other and form a pair of exceptional points in the present model $\tilde{H}_{\mathrm{BHZ}}(\boldsymbol{k})$; the edge spectrum becomes complex. Physically, the complex edge spectrum means the amplification (lasing) of the helical edge states. 
[1] V. V. Konotop, J. Yang, and D. A. Zezyulin, Nonlinear waves in $\mathcal{P} \mathcal{T}$-symmetric systems, Rev. Mod. Phys. 88, 035002 (2016).

[2] R. El-Ganainy, K. G. Makris, M. Khajavikhan, Z. H. Musslimani, S. Rotter, and D. N. Christodoulides, NonHermitian physics and PT symmetry, Nat. Phys. 14, 11 (2018).

[3] K. G. Makris, R. El-Ganainy, D. N. Christodoulides, and Z. H. Musslimani, Beam Dynamics in $\mathcal{P} \mathcal{T}$ Symmetric Optical Lattices, Phys. Rev. Lett. 100, 103904 (2008).

[4] S. Klaiman, U. Günther, and N. Moiseyev, Visualization of Branch Points in $\mathcal{P} \mathcal{T}$-Symmetric Waveguides, Phys. Rev. Lett. 101, 080402 (2008).

[5] A. Guo, G. J. Salamo, D. Duchesne, R. Morandotti, M. VolatierRavat, V. Aimez, G. A. Siviloglou, and D. N. Christodoulides, Observation of $\mathcal{P} \mathcal{T}$-Symmetry Breaking in Complex Optical Potentials, Phys. Rev. Lett. 103, 093902 (2009).

[6] C. E. Rüter, K. G. Makris, R. El-Ganainy, D. N. Christodoulides, M. Segev, and D. Kip, Observation of parity-time symmetry in optics, Nat. Phys. 6, 192 (2010).

[7] Z. Lin, H. Ramezani, T. Eichelkraut, T. Kottos, H. Cao, and D. N. Christodoulides, Unidirectional Invisibility Induced by $\mathcal{P} \mathcal{T}$-Symmetric Periodic Structures, Phys. Rev. Lett. 106, 213901 (2011).

[8] A. Regensburger, C. Bersch, M.-A. Miri, G. Onishchukov, D. N. Christodoulides, and U. Peschel, Parity-time synthetic photonic lattices, Nature 488, 167 (2012).

[9] L. Feng, Y.-L. Xu, W. S. Fegadolli, M.-H. Lu, J. E. B. Oliveira, V. R. Almeida, Y.-F. Chen, and A. Scherer, Experimental demonstration of a unidirectional reflectionless parity-time metamaterial at optical frequencies, Nat. Mater. 12, 108 (2013).

[10] B. Peng, Ş. K. Özdemir, F. Lei, F. Monifi, M. Gianfreda, G. L. Long, S. Fan, F. Nori, C. M. Bender, and L. Yang, Parity-timesymmetric whispering-gallery microcavities, Nat. Phys. 10, 394 (2014).

[11] J. Wiersig, Enhancing the Sensitivity of Frequency and Energy Splitting Detection by Using Exceptional Points: Application to Microcavity Sensors for Single-Particle Detection, Phys. Rev. Lett. 112, 203901 (2014).

[12] H. Hodaei, A. U. Hassan, S. Wittek, H. Garcia-Gracia, R. El-Ganainy, D. N. Christodoulides, and M. Khajavikhan, Enhanced sensitivity at higher-order exceptional points, Nature 548, 187 (2017).

[13] W. Chen, Ş. K. Özdemir, G. Zhao, J. Wiersig, and L. Yang, Exceptional points enhance sensing in an optical microcavity, Nature 548, 192 (2017).

[14] D. C. Brody and E.-M. Graefe, Mixed-State Evolution in the Presence of Gain and Loss, Phys. Rev. Lett. 109, 230405 (2012).

[15] T. E. Lee and C.-K. Chan, Heralded Magnetism in NonHermitian Atomic Systems, Phys. Rev. X 4, 041001 (2014).

[16] J. Li, A. K. Harter, J. Liu, L. de Melo, Y. N. Joglekar, and L. Luo, Observation of parity-time symmetry breaking transitions in a dissipative Floquet system of ultracold atoms, Nat. Commun. 10, 855 (2019).

[17] K. Kawabata, Y. Ashida, and M. Ueda, Information Retrieval and Criticality in Parity-Time-Symmetric Systems, Phys. Rev. Lett. 119, 190401 (2017)

[18] R. Hamazaki, K. Kawabata, and M. Ueda, Non-Hermitian Many-Body Localization, Phys. Rev. Lett. 123, 090603 (2019).
[19] L. Xiao, K. Wang, X. Zhan, Z. Bian, K. Kawabata, M. Ueda, W. Yi, and P. Xue, Observation of Critical Phenomena in ParityTime-Symmetric Quantum Dynamics, Phys. Rev. Lett. 123, 230401 (2019).

[20] Y. Wu, W. Liu, J. Geng, X. Song, X. Ye, C.-K. Duan, X. Rong, and J. Du, Observation of parity-time symmetry breaking in a single-spin system, Science 364, 878 (2019).

[21] K. Yamamoto, M. Nakagawa, K. Adachi, K. Takasan, M. Ueda, and N. Kawakami, Theory of Non-Hermitian Fermionic Superfluidity with a Complex-Valued Interaction, Phys. Rev. Lett. 123, 123601 (2019).

[22] M. Naghiloo, N. Abbasi, Y. N. Joglekar, and K. W. Murch, Quantum state tomography across the exceptional point in a single dissipative qubit, Nat. Phys. 15, 1232 (2019).

[23] N. Matsumoto, K. Kawabata, Y. Ashida, S. Furukawa, and M. Ueda, Continuous phase transition without gap closing in nonHermitian quantum many-body systems, arXiv:1912.09045.

[24] C. M. Bender and S. Boettcher, Real Spectra in Non-Hermitian Hamiltonians Having $\mathcal{P} \mathcal{T}$ Symmetry, Phys. Rev. Lett. 80, 5243 (1998); C. M. Bender, D. C. Brody, and H. F. Jones, Complex Extension of Quantum Mechanics, ibid. 89, 270401 (2002).

[25] A. Mostafazadeh, Pseudo-Hermiticity versus PT symmetry: The necessary condition for the reality of the spectrum of a non-Hermitian Hamiltonian, J. Math. Phys. 43, 205 (2002); Pseudo-Hermiticity versus PT-symmetry II: A complete characterization of non-Hermitian Hamiltonians with a real spectrum, 43, 2814 (2002); Pseudo-Hermiticity versus PT-symmetry III: Equivalence of pseudo-Hermiticity and the presence of antilinear symmetries, 43, 3944 (2002).

[26] N. Hatano and D. R. Nelson, Localization Transitions in Non-Hermitian Quantum Mechanics, Phys. Rev. Lett. 77, 570 (1996); Vortex pinning and non-Hermitian quantum mechanics, Phys. Rev. B 56, 8651 (1997).

[27] W. D. Heiss, The physics of exceptional points, J. Phys. A 45, 444016 (2012).

[28] Y. Ota, K. Takata, T. Ozawa, A. Amo, Z. Jia, B. Kante, M. Notomi, Y. Arakawa, and S. Iwamoto, Active topological photonics, Nanophotonics 9, 547 (2020).

[29] E. J. Bergholtz, J. C. Budich, and F. K. Kunst, Exceptional topology of non-Hermitian systems, arXiv:1912.10048.

[30] M. S. Rudner and L. S. Levitov, Topological Transition in a Non-Hermitian Quantum Walk, Phys. Rev. Lett. 102, 065703 (2009).

[31] M. Sato, K. Hasebe, K. Esaki, and M. Kohmoto, Time-reversal symmetry in non-Hermitian systems, Prog. Theor. Phys. 127, 937 (2012); K. Esaki, M. Sato, K. Hasebe, and M. Kohmoto, Edge states and topological phases in non-Hermitian systems, Phys. Rev. B 84, 205128 (2011).

[32] Y. C. Hu and T. L. Hughes, Absence of topological insulator phases in non-Hermitian $P T$-symmetric Hamiltonians, Phys. Rev. B 84, 153101 (2011).

[33] H. Schomerus, Topologically protected midgap states in complex photonic lattices, Opt. Lett. 38, 1912 (2013).

[34] S. Malzard, C. Poli, and H. Schomerus, Topologically Protected Defect States in Open Photonic Systems with Non-Hermitian Charge-Conjugation and Parity-Time Symmetry, Phys. Rev. Lett. 115, 200402 (2015).

[35] T. E. Lee, Anomalous Edge State in a Non-Hermitian Lattice, Phys. Rev. Lett. 116, 133903 (2016). 
[36] Q.-B. Zeng, B. Zhu, S. Chen, L. You, and R. Lü, Non-Hermitian Kitaev chain with complex on-site potentials, Phys. Rev. A 94, 022119 (2016).

[37] D. Leykam, K. Y. Bliokh, C. Huang, Y. D. Chong, and F. Nori, Edge Modes, Degeneracies, and Topological Numbers in NonHermitian Systems, Phys. Rev. Lett. 118, 040401 (2017).

[38] Y. Xu, S.-T. Wang, and L.-M. Duan, Weyl Exceptional Rings in a Three-Dimensional Dissipative Cold Atomic Gas, Phys. Rev. Lett. 118, 045701 (2017).

[39] H. Menke and M. M. Hirschmann, Topological quantum wires with balanced gain and loss, Phys. Rev. B 95, 174506 (2017).

[40] H. Shen, B. Zhen, and L. Fu, Topological Band Theory for Non-Hermitian Hamiltonians, Phys. Rev. Lett. 120, 146402 (2018); V. Kozii and L. Fu, Non-Hermitian topological theory of finite-lifetime quasiparticles: Prediction of bulk Fermi arc due to exceptional point, arXiv:1708.05841.

[41] K. Takata and M. Notomi, Photonic Topological Insulating Phase Induced Solely by Gain and Loss, Phys. Rev. Lett. 121, 213902 (2018).

[42] K. Kawabata, Y. Ashida, H. Katsura, and M. Ueda, Paritytime-symmetric topological superconductor, Phys. Rev. B 98, 085116 (2018).

[43] Z. Gong, Y. Ashida, K. Kawabata, K. Takasan, S. Higashikawa, and M. Ueda, Topological Phases of Non-Hermitian Systems, Phys. Rev. X 8, 031079 (2018).

[44] S. Yao and Z. Wang, Edge States and Topological Invariants of Non-Hermitian Systems, Phys. Rev. Lett. 121, 086803 (2018); S. Yao, F. Song, and Z. Wang, Non-Hermitian Chern Bands, ibid. 121, 136802 (2018).

[45] K. Kawabata, S. Higashikawa, Z. Gong, Y. Ashida, and M. Ueda, Topological unification of time-reversal and particle-hole symmetries in non-Hermitian physics, Nat. Commun. 10, 297 (2019).

[46] F. K. Kunst, E. Edvardsson, J. C. Budich, and E. J. Bergholtz, Biorthogonal Bulk-Boundary Correspondence in Non-Hermitian Systems, Phys. Rev. Lett. 121, 026808 (2018).

[47] K. Kawabata, K. Shiozaki, and M. Ueda, Anomalous helical edge states in a non-Hermitian Chern insulator, Phys. Rev. B 98, 165148 (2018).

[48] A. McDonald, T. Pereg-Barnea, and A. A. Clerk, PhaseDependent Chiral Transport and Effective Non-Hermitian Dynamics in a Bosonic Kitaev-Majorana Chain, Phys. Rev. X 8, 041031 (2018).

[49] C. H. Lee and R. Thomale, Anatomy of skin modes and topology in non-Hermitian systems, Phys. Rev. B 99, 201103(R) (2019).

[50] J. C. Budich, J. Carlström, F. K. Kunst, and E. J. Bergholtz, Symmetry-protected nodal phases in non-Hermitian systems, Phys. Rev. B 99, 041406(R) (2019).

[51] R. Okugawa and T. Yokoyama, Topological exceptional surfaces in non-Hermitian systems with parity-time and parityparticle-hole symmetries, Phys. Rev. B 99, 041202(R) (2019).

[52] T. Liu, Y.-R. Zhang, Q. Ai, Z. Gong, K. Kawabata, M. Ueda, and F. Nori, Second-Order Topological Phases in Non-Hermitian Systems, Phys. Rev. Lett. 122, 076801 (2019).

[53] T. Yoshida, R. Peters, N. Kawakami, and Y. Hatsugai, Symmetry-protected exceptional rings in two-dimensional correlated systems with chiral symmetry, Phys. Rev. B 99, 121101(R) (2019); K. Kimura, T. Yoshida, and N. Kawakami,
Chiral-symmetry protected exceptional torus in correlated nodal-line semimetals, ibid. 100, 115124 (2019).

[54] H. Zhou, J. Y. Lee, S. Liu, and B. Zhen, Exceptional surfaces in $\mathcal{P} \mathcal{T}$-symmetric non-Hermitian photonic systems, Optica 6, 190 (2019).

[55] F. K. Kunst and V. Dwivedi, Non-Hermitian systems and topology: A transfer-matrix perspective, Phys. Rev. B 99, 245116 (2019).

[56] K. Kawabata, K. Shiozaki, M. Ueda, and M. Sato, Symmetry and Topology in Non-Hermitian Physics, Phys. Rev. X 9, 041015 (2019).

[57] H. Zhou and J. Y. Lee, Periodic table for topological bands with non-Hermitian symmetries, Phys. Rev. B 99, 235112 (2019).

[58] W. B. Rui, Y. X. Zhao, and A. P. Schnyder, Topology and exceptional points of massive Dirac models with generic nonHermitian perturbations, Phys. Rev. B 99, 241110(R) (2019).

[59] K. Kawabata, T. Bessho, and M. Sato, Classification of Exceptional Points and Non-Hermitian Topological Semimetals, Phys. Rev. Lett. 123, 066405 (2019).

[60] K. Yokomizo and S. Murakami, Non-Bloch Band Theory of Non-Hermitian Systems, Phys. Rev. Lett. 123, 066404 (2019).

[61] P. A. McClarty and J. G. Rau, Non-Hermitian topology of spontaneous magnon decay, Phys. Rev. B 100, 100405(R) (2019).

[62] F. Song, S. Yao, and Z. Wang, Non-Hermitian Skin Effect and Chiral Damping in Open Quantum Systems, Phys. Rev. Lett. 123, 170401 (2019).

[63] E. J. Bergholtz and J. C. Budich, Non-Hermitian Weyl physics in topological insulator ferromagnet junctions, Phys. Rev. Research 1, 012003(R) (2019).

[64] C.-X. Guo, X.-R. Wang, C. Wang, and S.-P. Kou, NonHermitian dynamic strings and anomalous topological degeneracy on a non-Hermitian toric-code model with parity-time symmetry, Phys. Rev. B 101, 144439 (2020).

[65] B. Höckendorf, A. Alvermann, and H. Fehske, Non-Hermitian Boundary State Engineering in Anomalous Floquet Topological Insulators, Phys. Rev. Lett. 123, 190403 (2019).

[66] L. Herviou, N. Regnault, and J. H. Bardarson, Entanglement spectrum and symmetries in non-Hermitian fermionic noninteracting models, SciPost Phys. 7, 069 (2019).

[67] P.-Y. Chang, J.-S. You, X. Wen, and S. Ryu, Entanglement spectrum and entropy in topological non-Hermitian systems and nonunitary conformal field theory, Phys. Rev. Research 2, 033069 (2020).

[68] Q.-B. Zeng, Y.-B. Yang, and R. Lü, Topological phases in one-dimensional nonreciprocal superlattices, Phys. Rev. B 101, 125418 (2020).

[69] X.-X. Zhang and M. Franz, Non-Hermitian Exceptional Landau Quantization in Electric Circuits, Phys. Rev. Lett. 124, 046401 (2020).

[70] C. Poli, M. Bellec, U. Kuhl, F. Mortessagne, and H. Schomerus, Selective enhancement of topologically induced interface states in a dielectric resonator chain, Nat. Commun. 6, 6710 (2015).

[71] J. M. Zeuner, M. C. Rechtsman, Y. Plotnik, Y. Lumer, S. Nolte, M. S. Rudner, M. Segev, and A. Szameit, Observation of a Topological Transition in the Bulk of a Non-Hermitian System, Phys. Rev. Lett. 115, 040402 (2015).

[72] B. Zhen, C. W. Hsu, Y. Igarashi, L. Lu, I. Kaminer, A. Pick, S.-L. Chua, J. D. Joannopoulos, and M. Soljačić, Spawning rings of exceptional points out of Dirac cones, Nature 525, 354 (2015). 
[73] S. Weimann, M. Kremer, Y. Plotnik, Y. Lumer, S. Nolte, K. G. Makris, M. Segev, M. C. Rechtsman, and A. Szameit, Topologically protected bound states in photonic parity-time-symmetric crystals, Nat. Mater. 16, 433 (2017).

[74] L. Xiao, X. Zhan, Z. H. Bian, K. K. Wang, X. Zhang, X. P. Wang, J. Li, K. Mochizuki, D. Kim, N. Kawakami, W. Yi, H. Obuse, B. C. Sanders, and P. Xue, Observation of topological edge states in parity-time-symmetric quantum walks, Nat. Phys. 13, 1117 (2017).

[75] P. St-Jean, V. Goblot, E. Galopin, A. Lemaître, T. Ozawa, L. Le Gratiet, I. Sagnes, J. Bloch, and A. Amo, Lasing in topological edge states of a one-dimensional lattice, Nat. Photon. 11, 651 (2017).

[76] B. Bahari, A. Ndao, F. Vallini, A. El Amili, Y. Fainman, and B. Kanté, Nonreciprocal lasing in topological cavities of arbitrary geometries, Science 358, 636 (2017).

[77] G. Harari, M. A. Bandres, Y. Lumer, M. C. Rechtsman, Y. D. Chong, M. Khajavikhan, D. N. Christodoulides, and M. Segev, Topological insulator laser: Theory, Science 359, eaar4003 (2018); M. A. Bandres, S. Wittek, G. Harari, M. Parto, J. Ren, M. Segev, D. Christodoulides, and M. Khajavikhan, Topological insulator laser: Experiments, ibid. 359, eaar4005 (2018).

[78] A. Cerjan, S. Huang, K. P. Chen, Y. Chong, and M. C. Rechtsman, Experimental realization of a Weyl exceptional ring, Nat. Photon. 13, 623 (2019).

[79] H. Zhao, X. Qiao, T. Wu, B. Midya, S. Longhi, and L. Feng, Non-Hermitian topological light steering, Science 365, 1163 (2019)

[80] M. Brandenbourger, X. Locsin, E. Lerner, and C. Coulais, Non-reciprocal robotic metamaterials, Nat. Commun. 10, 4608 (2019); A. Ghatak, M. Brandenbourger, J. van Wezel, and C. Coulais, Observation of non-Hermitian topology and its bulkedge correspondence, arXiv:1907.11619.

[81] T. Helbig, T. Hofmann, S. Imhof, M. Abdelghany, T. Kiessling, L. W. Molenkamp, C. H. Lee, A. Szameit, M. Greiter, and R. Thomale, Generalized bulk-boundary correspondence in nonHermitian topolectrical circuits, Nat. Phys. 16, 747 (2020); T. Hofmann, T. Helbig, F. Schindler, N. Salgo, M. Brzezińska, M. Greiter, T. Kiessling, D. Wolf, A. Vollhardt, A. Kabaši, C. H. Lee, A. Bilušić, R. Thomale, and T. Neupert, Reciprocal skin effect and its realization in a topolectrical circuit, Phys. Rev. Research 2, 023265 (2020).

[82] L. Xiao, T. Deng, K. Wang, G. Zhu, Z. Wang, W. Yi, and P. Xue, Non-Hermitian bulk-boundary correspondence in quantum dynamics, Nat. Phys. 16, 761 (2020).

[83] S. Weidemann, M. Kremer, T. Helbig, T. Hofmann, A. Stegmaier, M. Greiter, R. Thomale, and A. Szameit, Topological funneling of light, Science 368, 311 (2020).
[84] W. P. Su, J. R. Schrieffer, and A. J. Heeger, Solitons in Polyacetylene, Phys. Rev. Lett. 42, 1698 (1979).

[85] A. Y. Kitaev, Unpaired Majorana fermions in quantum wires, Phys. Usp. 44, 131 (2002).

[86] B. A. Bernevig, T. L. Hughes, and S.-C. Zhang, Quantum spin Hall effect and topological phase transition in $\mathrm{HgTe}$ quantum wells, Science 314, 1757 (2006).

[87] C. L. Kane and E. J. Mele, Quantum Spin Hall Effect in Graphene, Phys. Rev. Lett. 95, 226801 (2005); $Z_{2}$ Topological Order and the Quantum Spin Hall Effect, 95, 146802 (2005).

[88] M. König, S. Wiedmann, C. Brüne, A. Roth, H. Buhmann, L. Molenkamp, X.-L. Qi, and S.-C. Zhang, Quantum spin Hall insulator state in HgTe quantum wells, Science 318, 766 (2007).

[89] C. W. J. Beenakker, Random-matrix theory of quantum transport, Rev. Mod. Phys. 69, 731 (1997); Random-matrix theory of Majorana fermions and topological superconductors, 87, 1037 (2015).

[90] R. Hamazaki, K. Kawabata, N. Kura, and M. Ueda, Universality classes of non-Hermitian random matrices, Phys. Rev. Research 2, 023286 (2020).

[91] S. Lieu, M. McGinley, and N. R. Cooper, Tenfold Way for Quadratic Lindbladians, Phys. Rev. Lett. 124, 040401 (2020).

[92] L. Sá, P. Ribeiro, and T. Prosen, Complex Spacing Ratios: A Signature of Dissipative Quantum Chaos, Phys. Rev. X 10, 021019 (2020).

[93] D. Bernard and A. LeClair, A classification of non-Hermitian random matrices, in Statistical Field Theories edited by A. Cappelli and G. Mussardo (Springer, Dordrecht, 2002), pp. 207-214.

[94] D. C. Brody, Biorthogonal quantum mechanics, J. Phys. A 47, 035305 (2014).

[95] D. V. Savin and V. V. Sokolov, Quantum versus classical decay laws in open chaotic systems, Phys. Rev. E 56, R4911(R) (1997).

[96] M. Hafezi, E. A. Demler, M. D. Lukin, and J. M. Taylor, Robust optical delay lines with topological protection, Nat. Phys. 7, 907 (2011); M. Hafezi, S. Mittal, J. Fan, A. Migdal, and J. M. Taylor, Imaging topological edge states in silicon photonics, Nat. Photon. 7, 1001 (2013).

[97] A. B. Khanikaev, S. H. Mousavi, W.-K. Tse, M. Kargarian, A. H. MacDonald, and G. Shvets, Photonic topological insulators, Nat. Mater. 12, 233 (2013).

[98] R. Süsstrunk and S. D. Huber, Observation of phononic helical edge states in a mechanical topological insulator, Science 349, 47 (2015).

[99] C. H. Lee, S. Imhof, C. Berger, F. Bayer, J. Brehm, L. W. Molenkamp, T. Kiessling, and R. Thomale, Topolectrical circuits, Commun. Phys. 1, 39 (2018). 